\title{
Desenvolvimento dialético de infraestrutura e superestrutura em plataformas livres para colaboração
}

\author{
Mateus F. L. Pelanda ${ }^{1}$, Frederick M.C. van Amstel ${ }^{1}$ \\ ${ }^{1}$ Departamento de Desenho Industrial - Universidade Tecnológica Federal do Paraná \\ (UTFPR) \\ Caixa Postal 80230-901 - Curitiba - PR - Brasil \\ mateuspelanda@alunos.utfpr.edu.br,vanamstel@utfpr.edu.br
}

\begin{abstract}
The development of Free/Libre and Open Source Software (FLOSS) for collaborative work present great challenges to interaction design, due to the inherent characteristics of collectivity, geographic distribution, and divergences. This research is conducting an ethnographic research in a collaborative project platform with the goal of understanding this kind of interaction design project. This platform is based on the Drupal framework and the Design Livre ideology. The preliminary results suggests that the design efforts are directed to the dialectics between infrastructure and superstructure.
\end{abstract}

Resumo. O desenvolvimento de plataformas Free/Libre and Open Source Software (FLOSS) para trabalho colaborativo oferece grandes desafios para o projeto de interação, devido às características de coletividade, distribuição geográfica e divergências. Com o objetivo de compreender esse tipo de projeto de interação, está sendo conduzida uma pesquisa etnográfica em um plataforma para projetos colaborativos desenvolvida à partir do framework Drupal e da ideologia do Design Livre. Os resultados preliminares da pesquisa sugerem que os esforços de projeto se orientam para a dialética entre infraestrutura e superestrutura.

\section{Introdução}

O desenvolvimento de plataformas FLOSS para trabalhos cooperativos auxiliados por computador (CSCW) geralmente é orientado por demandas de comunidades geodistribuídas que fazem uso de ferramentas e recursos disponíveis dentro da própria plataforma. Em diversos casos, este desenvolvimento se inicia com base em estruturas informais, caracterizadas por trabalhos paralelos, distribuídos, voluntariados e, na maioria das vezes, não remunerados [Simões 2010]. Neste contexto, os membros das comunidades colaboram a partir da utilização do sistema, realização de testes e com a execução de tarefas [Machado 2009]. Desta forma pode se entender que estas pessoas também projetam a plataforma, mas a partir de um "design pelo uso" [Dittrich, 2002], ou seja, ao utilizarem o sistema, também moldam interações que influenciam diretamente, a longo prazo, o projeto da própria plataforma.

Em uma perspectiva de longo prazo, as estruturas emergentes nesse sistema, criadas de maneira incremental por estes colaboradores, exigem a flexibilização do sistema, para que assim se mantenha uma colaboração constante. Desta forma os conflitos, que ocorrem entre os membros destas comunidades FLOSS, fazem, em diversos casos, que o planejamento e a formalização do sistema sejam substituídos pela cultura de improvisação, modificação espontânea dos objetos e "remendos" de códigos. Consequentemente, nestas comunidades, ocorrem diversas tensões e dificuldades na interação entre os colaboradores e desenvolvedores [Luke et al. 2004, Coleman 2005, Proulx and Couture 2006]. Ideologias são fundamentais para manter a coesão da comunidade em situações de conflito [Proulx and Couture 2006]. 
Ideologias podem ser definidas como sendo o conjunto de ideais compartilhados, de como funcionam ou deveriam funcionar determinados arranjos sociais [Eagleton 1991]. Desta forma, podem se fazer por relações imaginária entre indivíduos, assim caracterizando determinados grupos sociais. Sendo assim, a ideologia funciona como uma espécie de cola que preenche as fissuras no tecido social e mantém os participantes motivados apesar dos problemas técnicos enfrentados.

Existe uma lacuna sobre o papel da ideologia no projeto de interações que o estudo do desenvolvimento de plataformas livres pode ajudar a preencher. Como se dá, na prática, a reprodução ideológica e a reprodução de interações no desenvolvimento de plataformas livres para trabalho colaborativo? Para responder essa pergunta, primeiramente serão apresentados os conceitos de infraestrutura e superestrutura, bem como sua relação dialética.

\subsection{Infraestrutura e superestrutura}

Infraestrutura é um conjunto de relações materiais entre pessoas, tecnologias e espaços que suportam uma grande quantidade e diversidade de interações sociais [Jewett and Kling 1991]. Como estas interações são objeto de negociação política e econômica, infraestruturas estão sempre atreladas a superestruturas.

Superestrutura é uma estrutura que busca controlar interações sociais através de instâncias jurídicas e ideológicas [Althusser 1985]. Superestruturas são formadas, portanto, por infraestruturas de base, mas, possuem uma independência relativa a elas. Sendo assim, em uma perspectiva dialética, as infraestruturas moldam as superestruturas, enquanto as superestruturas moldam as infraestruturas [Althusser 1985]. Para compreender como isso acontece na prática, é importante adotar uma lente que permita reconhecer as ações individuais que constituem a produção coletiva tanto da infraestrutura, quanto da superestrutura.

\subsection{Ação infraestruturante e ação superestruturante}

A ação infraestruturante é a ação individual que visa a apropriação e criação de novas infraestruturas públicas, afim de prover novas interações e autonomia de uma determinada comunidade [Björgvinsson et al. 2010]. No caso de plataformas FLOSS, esse conceito explica porque as estruturas formais de um sistema se tornam emergentes e incrementais; elas seguem os desejos de interações futuras das comunidades que a mantém.

A ação infraestruturante também pode ser considerada uma ação de retorno, pois segue uma determinada ideologia sobre como deve funcionar a comunidade no futuro [Althusser 1985]. Essa ação retorna uma outra ação mais ampla, que denominaremos de ação superestruturante.

Já existem estudos que compreendem a ação infraestruturante no ambiente de desenvolvimento de plataformas FLOSS [e.g.Bossen et. al 2014, Haskel and Graham 2014, Haskel and Graham 2016, Haskel 2016, Unteidig 2017]. Também existem estudos sobre design de interação em ambientes de plataformas livres [e.g. van Amstel 2008, Corbucci 2011, Raza, Capretz and Ahmed 2012, Santos 2012, Saeed, Baywa and Mahmood 2015]. Porém, nenhum desses estudos conectam essas duas questões: ação infraestruturante e ação superestruturante.

\section{Caminho metodológico}

Esta pesquisa tem caráter exploratório e qualitativo e esta sendo conduzida por uma combinação dos métodos de etnografia de traço [Geiger \& Ribes 2011] e da etnografia de ações infraestruturantes [Karasti \& Blomberg 2018]. Este método está sendo aplicado aos registros públicos da concepção e desenvolvimento da plataforma Corais (corais.org). 
$\mathrm{Na}$ etnografia de traço os pesquisadores seguem e interpretam os vestígios de dados registrados na plataforma, com base na experiência interpretativa vivida e aprendida com determinada plataforma e comunidades digitais [Geiger and Halfaker 2017]. Através dos registros, os pesquisadores encontram pontos particularmente valiosos para a compreensão do estudo, uma vez que é o meio pelo qual as próprias comunidades distribuídas se comunicam e agem por meio delas [Geiger and Ribes 2011].

A seleção dos registros está sendo realizada com base na comunidade de desenvolvimento da plataforma corais, o grupo Metadesign (corais.org/metadesign). A fim de complementar tal documentação, também estão sendo investigados os arquivos do site Instituto de Design de Interação Faber Ludens (faberludens.com.br), por ser a instituição que deu origem a tal plataforma. A partir de tais registros, remontamos a narrativa principal presente no desenvolvimento da plataforma, a fim de auxiliar na compreensão e significação dos processos envolvidos [COSTA and GUALDA, 2010].

\section{Narrativa principal}

Design Livre é uma ideologia de projetos de design desenvolvida pelo Instituto FaberLudens [van Amstel et. al 2012] que possui foco em dar liberdade e independência aos "usuários", a fim de que os próprios membros de comunidades possam prover soluções para os próprios problemas. Para Gonzatto [2009], dentro da proposta do Design Livre, os "designers" transformam o status dos "usuários" em "designers", diferente do Design Participativo, qual o designer projeta com os "usuários", e do Design Centrado no Usuário, que o "designer" projeta para os "usuários". Sendo assim, se torna necessário adotar uma documentação aberta, compartilhando o método de desenvolvimento, modelos mentais, prototipagem, testes e demais decisões de design [Meireles 2011].

A plataforma Corais foi desenvolvida em 2011 por alunos e professores do Instituto Faber Ludens para disseminar e suportar a ideologia do Design Livre, a partir da utilização do Open Atrium (drupal.org/project/openatrium), uma plataforma de códigos abertos baseada em módulos do Drupal (drupal.org). Na plataforma, grupos e coletivos podem utilizar ferramentas livres para se organizar e colaborar. O processo de desenvolvimento e de atualizações do software se deram a partir de demandas emergentes por próprios membros dos grupos que utilizam a plataforma. Estas pessoas conceberam possíveis soluções e melhorias com base nas necessidades e problemas vivenciados, durante a utilização das ferramentas disponibilizadas. Estas necessidades foram demonstradas por meio de comentários no grupo Metadesign, as quais eram priorizadas através de processos de votação o que poderia ser implementado. A partir desses requisitos, conforme a relevância e disponibilidade de módulos do Drupal, os desenvolvedores da plataforma implementaram no software melhorias.

A correção de "bugs" e a implementação de novas features ocorriam em tempo real, sem ser previamente planejado, fazendo com que a plataforma nunca estivesse finalizada, a colocando em um patamar de eterno protótipo. Se algo ocorresse de maneira errada, ou a ferramenta/interação não era útil para toda a comunidade, ou atendida somente alguma demanda unitária, tal implementação era descontinuada e se voltava a versão anterior a tal atualização.

\subsection{Análise em andamento}

A intenção de existir uma abertura para o desenvolvimento do espaço coletivo e os registros públicos deste processo na plataforma, serviram como ações infraestruturantes, pois auxiliaram no desenvolvimento de políticas libertárias para a plataforma e para a criação coletiva. Destas também emergiram novas ações superestruturantes, ao servirem como exemplo para os grupos de como utilizarem estes processos para as próprias necessidades. 
A plataforma e suas ferramentas são objetos em constante movimento, com qualidades emergentes, necessitando de constantes modificações e mudanças, assim necessitando da abertura das caixas pretas (modificação dos módulos do drupal), para se repensar tanto nas interações que ocorriam entre estes módulos, quanto das interações sociais que ocorriam dentro da plataforma. Estas serviram como uma forma de criar interações humano-humano, por meio de interações humano-computador.

Os módulos eram utilizados como "peças de um quebra-cabeça", a fim de moldar como seria formado o software, assim constituindo uma "colcha de retalhos", um "patchwork de gambiarras" que cumprem as funções para o funcionamento da plataforma e das interações dessa. O problema criado com a utilização desses módulos é a herança das infraestruturas e das características do Drupal, assim como da dependência a outra plataforma.

Durante o desenvolvimento dos projetos, com o compartilhamento livre e aberto dos processos de design, ocorreram ações superestruturantes, tal como a escrita coletiva dos livros Design Livre (2012) e Coralizando (2014), a publicação de métodos de design no wiki da plataforma, a realização de palestras em eventos acadêmicos e profissionais e a escrita de artigos acadêmicos.

\subsection{A dialética do desenvolvimento de plataformas livres}

A partir da análise realizada, até o presente momento, foi possível generalizar as ações infraestruturantes e ações superestruturantes na formação do projeto de interações da plataforma. O desenvolvimento se fez por meio de uma relação dialética, da qual a implementação e aperfeiçoamento das ferramentas se realizava pela síntese entre demandas internas do software e relevância dos problemas (concebidas através do uso constante da das ferramentas disponíveis), com a capacidade produtiva de combinar e implementar diferentes módulos do Drupal. Ao mesmo tempo, enquanto ocorriam as ações infraestruturantes no desenvolvimento da plataforma, estas exerciam influência nas ações superestruturantes da ideologia do Design Livre. A abertura e compartilhamento do código de design, influenciou na mudança de status dos usuários para a categoria de designers, assim incluindo novos participantes e fomentando novos projetos, de maneira cíclica. Desta forma, este modelo histórico do projeto de interação da plataforma se encontra em um ciclo iterativo, conforme a Figura 1. Esse ciclo serve tanto para manter quanto para transformar a comunidade que utiliza a plataforma.

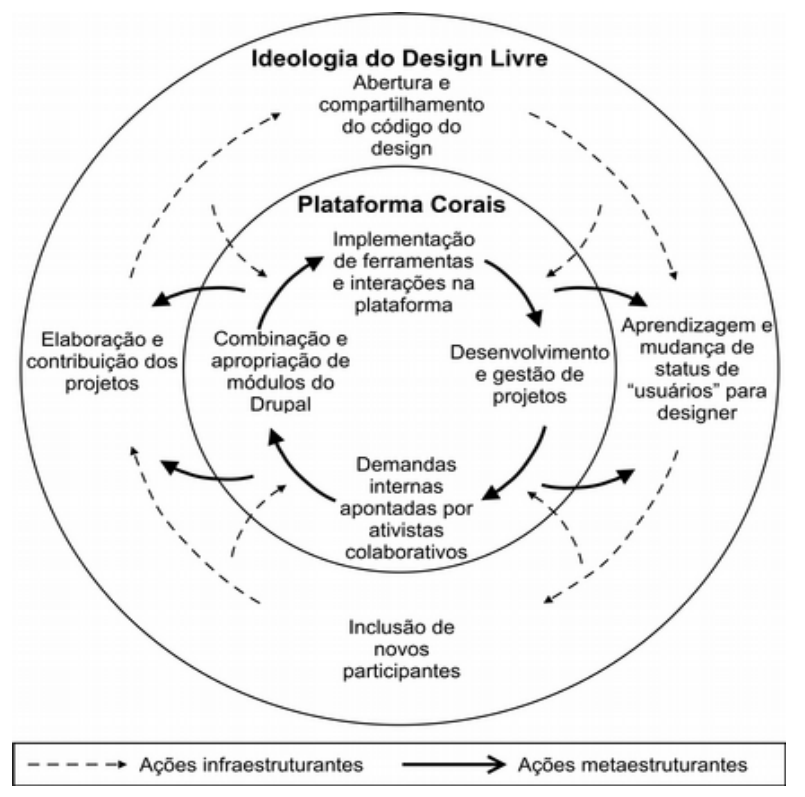

Figure 1. Ciclo de ações infraestruturantes e superestruturantes vinculadas ao desenvolvimento da plataforma Corais. 


\section{Discussão}

No que se refere ao desenvolvimento FLOSS, as ideologias e superestruturas se fazem tão importante quanto as estruturas e ações infraestruturantes que as moldam. Interpretar como estas agem nos processos de desenvolvimento a longo prazo destas plataformas, torna possível explicar empiricamente como a ideologia se materializa na prática da apropriação tecnológica. Em plataformas livres para CSCW, o design de interação ocorre de forma colaborativa e distribuída, tendo influências diretas das ideologias de projetos, compartilhada pelos membros dos grupos, na formação de infraestruturas técnicas e culturais. O desenvolvimento da plataforma Corais, por exemplo, está atrelado ao desenvolvimento da ideologia do Design Livre.

Acreditamos que a partir do desenvolvimento deste estudo, estaremos contribuindo com a literatura de design participativo com a conceitualização da dialética entre superestrutura e infraestrutura, bem como a distinção da ação superestruturante da ação infraestruturante. Espera-se, com isso, demonstrar a importância da ideologia nos estudos de práticas de desenvolvimento e design de plataformas.

\section{Referências}

Althusser, L. (1985). “Aparelhos Ideológicos de Estado”. Graal, Rio de Janeiro.

Björgvinsson, E., P. Ehn, and P-A. Hillgren. (2010). "Participatory design and "democratizing innovation"”, In: Proceedings of the 11th Biennial Participatory Design Conference (PDC '10).

Bossen, C., Ehn, P., Karasti, H., Di Salvo, C., Clement, A., Pipek, V. and Dittrich, Y. (2014). "Infrastructuring, collaboration and evolving socio-material practices of changing our world", In: Proceedings of the 13th Participatory Design Conference on Short Papers, Industry Cases, Workshop Descriptions, Doctoral Consortium Papers, and Keynote Abstracts - PDC '14 - Volume 2. doi:10.1145/2662155.2662211

Coleman, G. (2005). “The Politics of Open Source Adoption, NGO's in the Developing World.”, In: SSRC Report for the Tactical Tech Collective.

Corbucci, H. (2011). "Métodos ágeis e software livre: um estudo da relação entre estas duas comunidades". Dissertação de Mestrado, Instituto de Matemática e Estatística da Universidade de São Paulo. Disponível em: https://github. com/hugocorbucci/dissertacao-mestrado. Acesso em: 20 junho 2019.

COSTA, G.M.C. and GUALDA, D.M.R. (2010). "Antropologia, etnografia e narrativa: caminhos que se cruzam na compreensão do processo saúde-doença". História, Ciências, Saúde - Manguinhos, Rio de Janeiro, v.17, n.4. p.925-937.

Dittrich Y., Eriksén S. and Hansson C. (2002). "PD in the Wild: Evolving practices of Design in Use". In: Participatory Design Conference. Malmö, Sweden.

Eagleton, T. (1991). "Ideology:An Introduction”. Verso, New York.

Geiger, R. S. and Halfaker, A. (2017). "Operationalizing Conflict and Cooperation Between Automated Software Agents in Wikipedia: A Replication and Expansion of 'Even Good Bots Fight'”, In: Proc. ACM Hum.-Comput. Interact. 1, CSCW, Article 49 (Dec. 2017), 33 pages. https://doi.org/10.1145/3134684

Geiger, R.S. and Ribes, D. (2011). "Trace Ethnography: Following Coordination Through Documentary Practices"., In: Proc HICSS. IEEE.

Gonzatto, R. F. (2009). "Design Livre: processo aberto, desenvolvimento liberto", http://www.gonzatto.com/design-livre-processo-aberto/. Acesso em: 15 maio 2019.

Haskel, L. F. (2016). "Participatory design and free and open source software in the not for profit sector: the Hublink Project". Doctor Thesis. Bournemouth University, 
Centre for Digital Entertainment.

Haskel, L. and Graham, P. (2014). "Hublink: a case study in participatory design and open source in the third sector", In: Proceedings of the 13th Participatory Design Conference: Short Papers, Industry Cases, Workshop Descriptions, Doctoral Consortium papers, and Keynote abstracts- Volume 2. ACM, 2014. DOI: $10.1145 / 2662155.2662237$

(2016). "Whats GNU got to do with it? Participatory Design, Infrastructuring and Free/Open Source Software", In: Proceedings of the 14th Participatory Design Conference: Short Paper. ACM, 2016. doi:10.1145/2948076.2948090

Jewett, T. and Kling, R. (1991). "The dynamics of computerization in a social science research team: A case study of infrastructure, strategies, and skills", In: Social Science Computer Review 9(2): 246-275.

Karasti, H. and Blomberg J. (2018). "Studying Infrastructuring Ethnographically", In: Computer Supported Cooperative Work (CSCW), vol. 27, no. 2. (Special issue on 'Infrastructuring and Collaborative Design').

Luke, R., Clement, A., Terada, R., et al. (2004), "The promise and perils of a participatory approach to developing an open source community learning network.", In: Proceedings of the Participatory Design Conference 2004: Artful Integration: Interweaving Media, Materials, and Practices, Toronto, ON, pp. 11-19.

Machado, M. B. (2009) "Distros e comunidades: a dinâmica interna de Debian, Fedora, Slackware e Ubuntu", In: Aguiar, V. M. (Org.). Software Livre, Cultura Hacker e Ecossistema da Colaboração. São Paulo: Momento Editorial.

Meireles, A. V. (2011). "A inovação no Design de Interação: Design Livre para modelos sustentáveis.", Trabalho de Conclusão do Curso. Especialização em Design de Interação, PUC Minas, Belo Horizonte.

Poulx, S. and Couture, S. (2006). "Práticas de cooperação e ética da partilha na intersecção de dois mundos sociais: militantes do software livre e grupos comunitários no Quebeque.”, In: Análise Social, vol. XLI (181), 1057-1074.

Raza A.; Capretz L. F. and Ahmed F. (2012). "An open source usability maturity model (OS-UMM)”, In: Comput Hum Behav 28(4):1109-1121

Santos, A. P. O. (2012). "Aplicação de práticas de usabilidade ágil em software livre". São Paulo: IME-USP, 2012. 131 p. Dissertação (Mestrado) - Mestrado em Ciência da Computação, Universidade São Paulo, São Paulo.

Saeed, S., Baywa, I. S. and Mahmood, Z. (2015). "Human Factors in Software Development and Design", In: IGI Global, USA.

Simões, S. M. D. (2010). "Metodologia de avaliação de aplicações para descrição de arquivo definitivo". Lisboa: [s. n.]. Tese de Mestrado em Ciências da Documentação e Informação. Biblioteconomia e Documentação. Universidade de Lisboa.

Unteidig A., Cobreros B. D., Calderon-Lüning E. and Joost G. (2017). "Digital commons, urban struggles and the role of Design", In: The Design Journal, 20:sup1, S3106-S3120, DOI: 10.1080/14606925.2017.1352818

van Amstel, F. M. (2008). "Das Interfaces às Interações: design participativo do Portal BrOffice.org”. Dissertação de Mestrado em Tecnologia - Programa de PósGraduação em Tecnologia, Curitiba: UTFPR.

van Amstel, F. M. C. et al. (2012). "Design Livre”. Sao Paulo: Clube dos Autores. 